\title{
Decision-making in the Internet age: opportuni- ties to reshape our role in the OR and beyond
}

Alejandro R. Jadad MD DPHIL FRCPC

M

OST anesthesiologists practicing today are likely to belong to the generation that witnessed the birth of the personal computer, smart mobile communication devices, wireless networks, the Internet and the World Wide Web. During the past decade, these information and communication technologies (ICTs) have triggered a dramatic transformation of major industries. In fact, banking, entertainment and tourism will probably never be the same now that online consumer services have been introduced. What effect are these ICTs having on the practice and teaching of medicine? What will be their impact on the future of anesthesia?

In this session, participants will learn about:

- Leading organizations and initiatives that are promoting major transformation of the health system through the use of ICTs;

- New and emerging ICTs that could be used by anesthesiologists to improve the way in which they make decisions in the operating room and beyond;

- Efforts to overcome current barriers for the use of the Internet and other ICTs in busy clinical environments; and

- The impact that ICTs could have on the role of anesthesiologists within the health system, and on their relationship with other health professionals and the public.

Dr. Jadad is the Canada Research Chair in eHealth Innovation and the Rose Family Chair in Supportive Care at the Centre for Global eHealth Innovation; Departments of Anesthesia, and Health Policy, Management and Evaluation; University Health Network and University of Toronto, Toronto, Ontario, Canada.

Address correspondence to: Dr. Alejandro R. Jadad, Centre for Global eHealth Innovation, R. Fraser Elliott Building, 4th Floor, Toronto General Hospital, 190 Elizabeth Street, Toronto, Ontario M5G 2C4, Canada. Phone: 416-340-4800, ext. 6903; Fax: 416-340-3595

E-mail: ajadad@ehealthinnovation.org

CAN J ANESTH $2005 / 52: 6 /$ pp Rl 